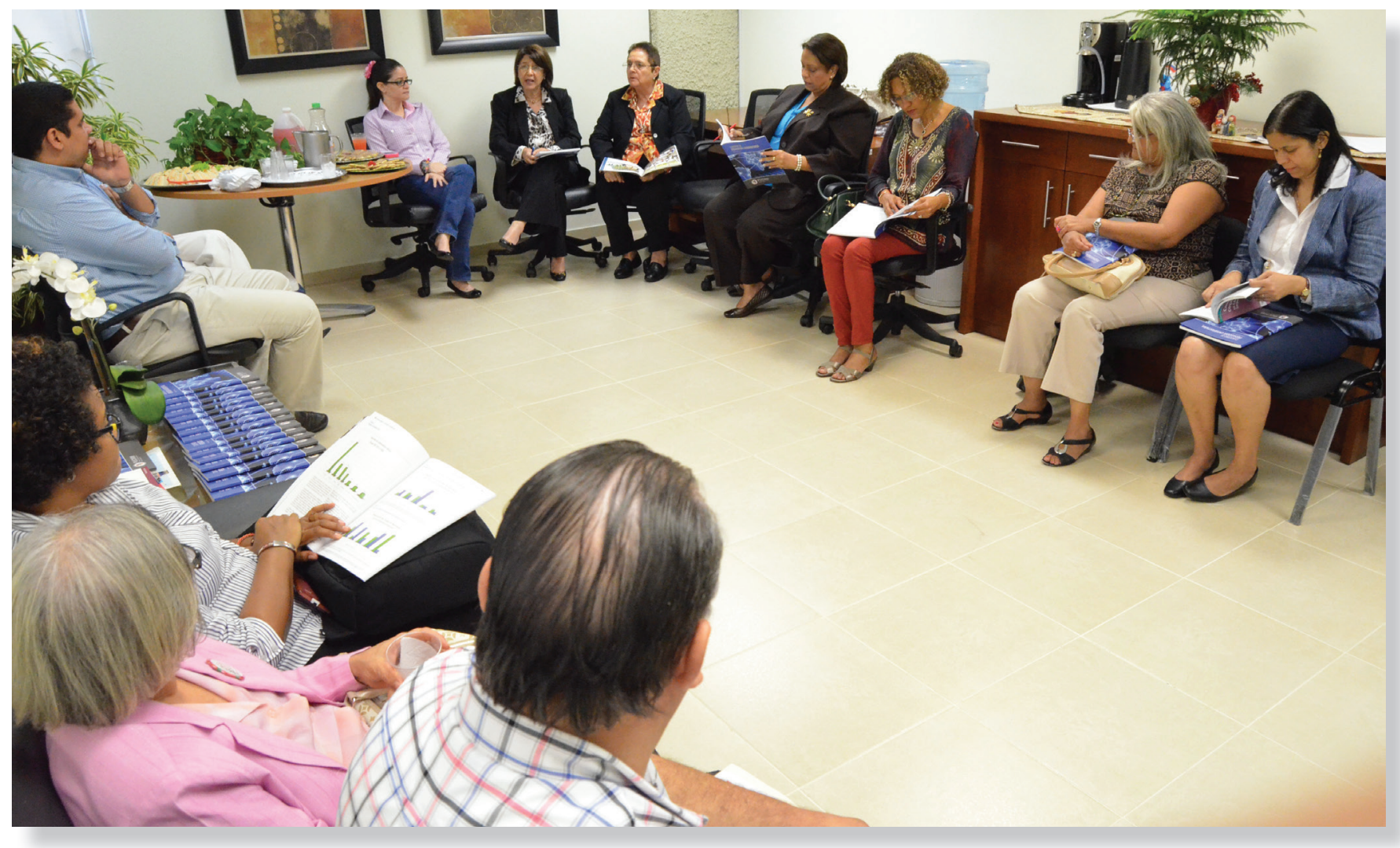

\title{
La XV peña pedagógica
}

Estos encuentros, de carácter informal entre el profesorado de la PUCMM, se organizan para compartir ideas y opiniones de los artículos publicados en la última edición del Cuaderno de Pedagogía Universitaria (CPU). En esta ocasión se reseña la edición número XXII, correspondiente a julio-diciembre 2014. Las reuniones se llevaron a cabo el martes 01 y el miércoles 02 de diciembre de 2015, en el campus Santiago y Santo Tomás de Aquino, respectivamente. A continuación se presentan las ideas principales del encuentro.

a profesora Ana Margarita Haché dio la bienvenida a los asistentes. Manifestó que con La XV Peña se celebraban 10 años de la instauración del CPU, el cual surgió como una formación alternativa, a través de la lectura y la escritura, para que los profesores que no podían asistir a los cursos presenciales pudieran nutrirse de las investigaciones que se publican en el Cuaderno. Valoró los logros que se han tenido durante estos diez años de publicación, en especial la versión en línea. Durante la actividad se realizó una demostración de cómo el nuevo sistema de OJS, permite una mayor visibilidad de los artículos gracias a su conexión a varios buscadores de información. Se explicó la dinámica que se utilizaría para compartir los artículos.

\section{Campus Santiago}

La articulista Gisela León compartió parte de su experiencia de investigación en el artículo "Innovación en el proceso de enseñanza-aprendizaje por competencias de la asignatura Análisis de Estados Financieros", afirmando que lo que marcó la diferencia para ella y su compañera, la profesora Rosanna Crespo, fue el acompañamiento 
que recibieron durante el proceso. Aseguró que sus objetivos no eran solamente realizar el cambio por competencias de la asignatura Análisis de Estados Financieros, sino de impactar a través de ella otras asignaturas de la carrera de Gestión Financiera y Auditoria; atestiguó que gracias a la investigación se realizaron reuniones con sus homólogos del Departamento en un intento de armonizar las asignaturas sobre las competencias que consideraron importantes para el desarrollo integral de los estudiantes en la parte ética y financiera.

Por su parte, la profesora Miriam Valdez comentó el artículo que escribió junto al profesor Aldemaro Muñiz "Uso de las TIC en la Práctica Pedagógica de los Docentes del Departamento de Ciencias Jurídicas"; alegó gran preocupación por el uso de la tecnología en la docencia específicamente en el uso de las herramientas Materias en Línea y de la Plataforma Virtual de Aprendizaje (PVA). Adujo que la PVA es utilizada como medio para el envío de tareas; pero que debería aprovecharse como medio para propiciar debates, compartir videos y permitir la interacción mediante los foros de aprendizaje. Además, la articulista argumentó que probablemente los profesores no hacen uso de la PVA, porque requiere tiempo y esfuerzo que no son contabilizados como horas de docencia.

La profesora Claudia Jerez, del Departamento de Medicina, asintió con la opinión de la articulista, añadiendo que algunos estudiantes se quejan de las horas que tienen que pasar en línea y que además deben cumplir con el horario de clases presenciales, consideró que cada vez más la Universidad debe inclinarse a complementar la enseña a través de la creación de entornos virtuales de aprendizaje.

La vicerrectora académica, campus Santiago, Rafaela Carrasco, estimó como una tarea pendiente de la vicerrectoría legislar la valoración del tiempo que invierten los docentes en el diseño de sus clases para los entornos virtuales. Asimismo el rector, padre Alfredo de la Cruz, motivó a los docentes a combinar los estilos de enseñanza que puedan ser útil para responder a las necesidades de aprendizaje de los estudiantes mientras se logra materializar el cambio.

Los articulistas Hamlet Castillo, Antonio Rivero y Wanda Sepúlveda, expusieron ante los presentes su artículo "Desarrollo por Competencias de la Asignatura Matemática Universitaria I". Rivero reveló los resultados que han tenido después de la investigación considerando como reto principal lograr que las Matemáticas cobraran vida para los estudiantes, afirmó que gracias al enfoque por competencias, no solo pudieron potencializar el conocimiento matemático, sino que además, desarrollaron el pensamiento analítico y lógico. En este sentido, Castillo sostuvo que cuando integran las ecuaciones y los conceptos a situaciones de la vida diaria, los estudiantes valoran el proceso y entienden la importancia de las Matemáticas. Sepúlveda añadió que algunos docentes muestran resistencia para trabajar por competencias, explica, que hace falta un cambio de actitud ante el enfoque. Gracias al trabajo de los articulistas la asignatura Matemática Universitaria está siendo impartida por competencias.

La profesora Ana Margarita Haché valoró los aportes de cada uno de los articulistas, agradeció la asistencia de los presentes y despidió el encuentro.

Asistentes a la Peña: P. Alfredo de la Cruz, Ana Margarita Haché, Antonio Rivero, Ariosto Díaz, Carmen Luisa González, Claudia Jerez, Elsa Margarita Olivo, Gisela León, Gladys Rodríguez, Florangel Mata, Francis Núñez, Hamlet Castillo, Idelise Sagredo, llona de la Rocha, José Guillermo Marte, Lilian García de Brens, Liliana Montenegro, Luvian Corniel, Luz Eneida Rodríguez, Manuel Maza, Margie Sánchez, María Irene Danna, María Luisa Tavárez, Marialys Santana, Mary Ann Butler, Mayra Leonard, Mayra Ruiz, Mélida Rosa, Miriam Valdez, Nora Ramírez, Omar Beltré, Rafael Oscar Madera, Rafaela Carrasco, Roberto Rodríguez, Rosa Bertilia Rivero, Wanda Sepúlveda y Yuberkis Cruz.

\section{Campus Santo Tomás de Aquino}

El primer artículo en ser compartido fue "Retos formativos y pedagógicos del doctorado en el siglo XXl" del profesor Enrique Sánchez, quien aprovechó para compartir con los presentes las innovaciones del Doctorado en Español, el cual está siendo impartido desde nuestra Universidad. Es el primer doctorado cuyo programa ha sido generado e impartido por profesores que residen en la República Dominicana. Por otra parte, motivó a los docentes presentes a que sus intereses pedagógicos fueran convergentes con sus intereses investigadores, de esta manera pueden aprovechar sus prácticas para investigación y estas a su vez, se pueden convertir en publicaciones. Valoró la escritura académica, afirmando que son aportes que se hacen a la ciencia desde cada una de las distintas disciplinas.

La profesora Ana Margarita Haché, resaltó el compromiso social de la Universidad mediante la creación de planes de estudios que respondan a las necesidades de la sociedad dominicana. En este mismo orden, la profesora Oliva Hernando sostuvo que la Universidad se rige en base al humanismo cristiano y en base al Modelo Educativo, siempre siendo fiel a su identidad y a sus principios.

El profesor Antonio Espín resaltó la importancia de realizar cambios políticos en nuestra sociedad, afirmando que es tiempo de otorgar primacia a la 
educación; de manera que se dé prioridad a la profesionalidad de los agentes que manejan las instituciones del Estado.

Por su parte, la profesora Jeanette Chaljub explicó que el artículo "Trabajo Colaborativo como estrategia de Enseñanza en la Universidad" es un resumen de su libro y tesis doctoral sobre el tema. Compartió algunas de las técnicas para llevar a cabo la metodología dentro de las aulas y los diferentes tipos de trabajo que surgen desde el enfoque; añadió que trabajar con este método implica un seguimiento continuo por parte del profesor, una rúbrica de evaluación clara y la evaluación de la metodología.

La profesora Rosa Noyola, cuestionó a la autora sobre las competencias que se desarrollan a partir del trabajo colaborativo. Chaljub manifestó que las principales son el pensamiento crítico, la lectura crítica, desarrollo de relaciones interpersonales, respeto a la diversidad de opiniones, la argumentación, la investigación y el pensamiento complejo.

La profesora Aida Consuelo Hernández cuestionó sobre la reacción de los estudiantes ante la nueva forma de trabajo. La autora afirmó que los estudiantes se sienten seguros, cuando conciben que están siendo guiados y la metodología de evaluación se les transparenta desde el primer día de clases. Chaljub recalcó que el Trabajo Colaborativo no debe ser utilizado como la única técnica en el proceso de enseñanza-aprendizaje.

En otro tenor, Chaljub señaló la necesidad de la creación de una cultura de liderazgo compartido entre los docentes, donde el compañerismo permita crear comunidades de aprendizaje. El profesor Sánchez, puntualizó que estas comunidades son un reto, sobre todo en el área de humanidades. Estimó como una oportunidad para incentivar estas sociedades los grupos de investigación que busquen un beneficio común.

Florilena Paredes habló sobre la reseña del libro Competencias comunicativas en Educación Superior y motivó a los presentes a utilizar la biblioteca pedagógica del Centro de Desarrollo Académico para mejorar sus prácticas docentes.

El articulista Johannes Olsen autor del artículo "Maestrías en Periodoncia e Implantología Oral y Prostodoncia e Implantología: una mirada desde dentro al proceso de enseñanza-aprendizaje" compartió con los presentes su experiencia como estudiante de la Maestría Periodoncia e Implatología Oral. Valoró la implementación de la teoría a la práctica, los instrumentos, la riqueza del currículo, el tratamiento multidisciplinario de los pacientes, así como la ética profesional que se exhiben desde los planes de estudios de ambas maestrías.

Finalmente, la profesora Ana Margarita Haché señaló algunos de los objetivos para las nuevas ediciones del CPU. Se clausuró el evento dando las gracias a los presentes por su asistencia y aportes.

Asistentes a la Peña: Ana Margarita Haché, Aida Consuelo Hernández, Andrés Mota, Antonio Espín, Asela Peguero, Bárbara Campos, Clara Echevarría, Clara Cruz, Giovanna Cabreja, Juliana De los Santos, Félix Rodríguez, Florangel Mata, Florilena Paredes, Francisco Arias, Inmaculada Torres, Jesús Michelén, Larissa Madera, Lilian Castillo, Michel Piña de Rosario, Oliva Hernando, Patricia Tineo, Rosa Noyola, Rubén Estrella y Salvador Montás.

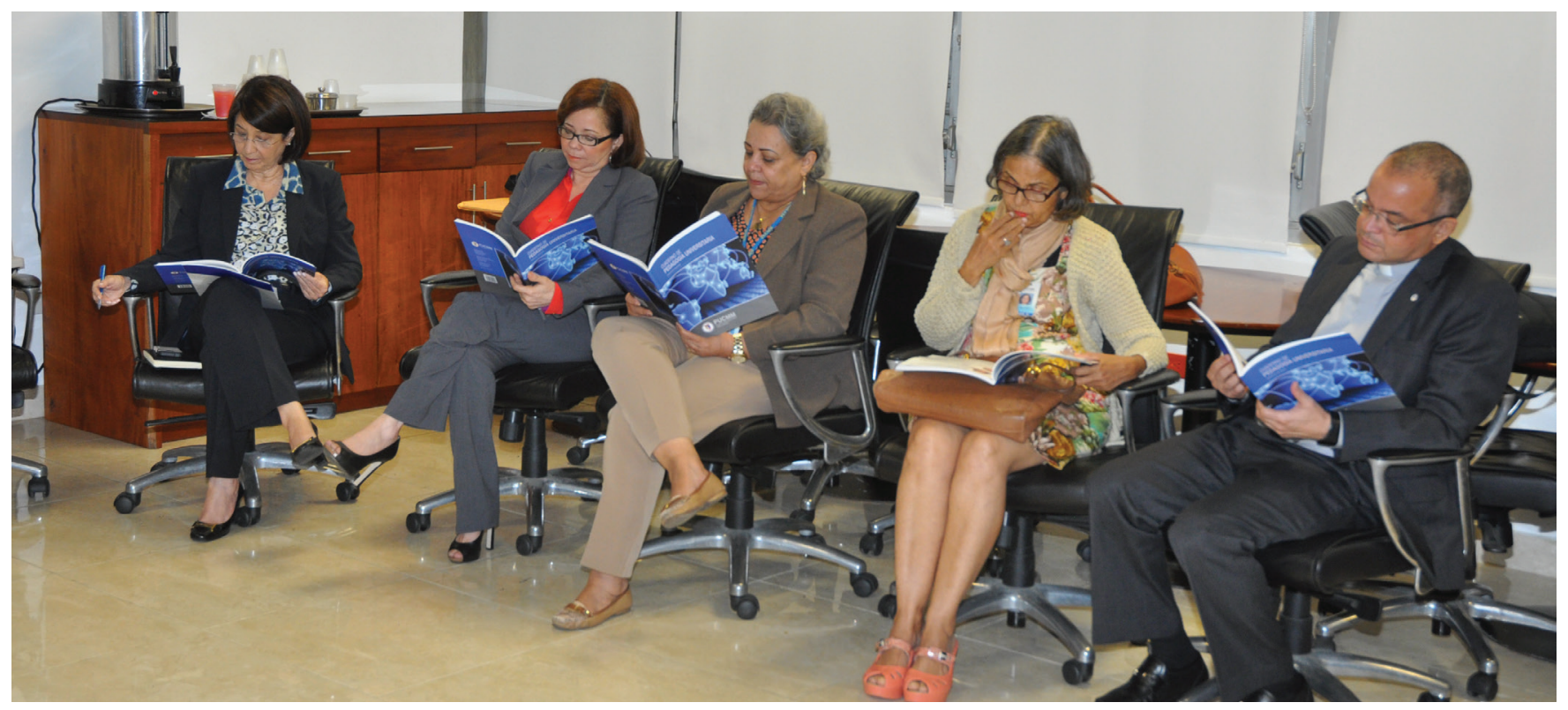

